\title{
Spread of the SARS-CoV-2 UK-variant in the South East of France: impact on COVID-19 patients age, comorbidity profiles and clinical presentation
}

Johan Courjon ( $\nabla$ courjon.j@chu-nice.fr )

Université Côte d'Azur, Inserm, U1065, C3M, Nice

Julie Contenti

Université Côte d'Azur, CHU Nice

Elisa Demonchy

Université Côte d'Azur, CHU Nice

Jacques Levraut

Université Côte d'Azur, CHU Nice

Pascal Barbry

Institut de Pharmacologie Moleculaire et Cellulaire, UMR7275 CNRS/UNS

Géraldine Rios

Institut de Pharmacologie Moleculaire et Cellulaire, UMR7275 CNRS/UNS

Jean Dellamonica

Université Côte d'Azur, CHU Nice

David Chirio

Université Côte d'Azur, CHU Nice

Caroline Bonnefoy

Université Côte d'Azur, CHU Nice

Valérie Giordanengo

Université Côte d'Azur, Inserm, U1065, C3M, Nice

Michel Carles

Université Côte d'Azur, CHU Nice

\section{Research Article}

Keywords: SARS-CoV-2, COVID-19, 20I/501Y.V1, SARS-CoV-2 variants

Posted Date: March 30th, 2021

DOI: https://doi.org/10.21203/rs.3.rs-343317/v1 
License: (c) (i) This work is licensed under a Creative Commons Attribution 4.0 International License. Read Full License 


\section{Abstract}

Objectives: The variant 201/501Y.V1, associated to a higher risk of transmissibility, emerged in Nice city (South East of France, French Riviera) during January 2021. The pandemic has resumed late December 2020 in this aera. A high incidence rate together with a fast turn-over of the main circulating variants, provided us the opportunity to analyze modifications in clinical profile and outcome traits.

Methods: Observational study in the University hospital of Nice from December 2020 to February 2021. We analyzed data of sequencing of SARS-CoV-2 from the sewage collector and PCR screening from all positive samples at the hospital. Then, we described the characteristics of all COVID-19 patients admitted in the emergency department (ED) $(n=1247)$ and those hospitalized in the infectious diseases ward or ICU $(n=232)$.

Results: the UK-variant was absent in this area in December, then increasingly spread in January representing $59 \%$ of the PCR screening performed mid-February. The rate of patients over 65 years admitted to the ED decreased from $63 \%$ to $50 \%(p=0.001)$. The mean age of hospitalized patients in the infectious diseases ward decreased from 70.7 to $59.2(p<0.001)$ while the proportion of patients without comorbidity increased from $16 \%$ to $42 \%(p=0.007)$.

Conclusion: Spread of the UK-variant in the South East of France affects younger and healthier patients.

\section{Introduction}

Starting in September 2020, the new SARS-CoV-2 variants called VOC-202012/1 (lineage B.1.1.7, 20I/501Y.V1 or GR/501Y.V1) have rapidly spread in the UK, associated to a higher risk of transmissibility 1. A matched cohort study including 109812 patients in the UK showed an increase in deaths from 2.5 to 4.1 per 1000 cases detected associated to the 20I/501Y.V1 variant ${ }^{2}$.

The spread of this variant (201/501Y.V1 i.e. "UK-variant") in continental Europe was identified late December 2020, as reported by the European Center for Disease Prevention and Control (ECDC) with the first reports in Netherland, Belgium, Denmark ${ }^{3}$. It was reported in France late December 2020 4,5.

After a second outbreak in November (with an incidence up to 501/100000 inhabitants), the pandemic declined in France in December 2020. At December 10th, the administrative area around Nice, French Riviera, in the South East of France, which totalizes more than 1 million inhabitants, exhibited a stable incidence of new positive cases at $169 / 100,000$, i.e. a slightly higher value relative to the whole French territory. Contrary to the rest of France, the pandemic has resumed in this area since late December, reaching an incidence of $583 / 100,000$ on February 13 th ${ }^{5}$.

At the same time, the UK-variant, emerged in January in our area. The specific epidemiologic trends of COVID-19 epidemic in our area, i.e. a high incidence rate together with a fast turn-over of the main 
circulating variants, provided us the opportunity to analyze the UK-variant-related clinical profile and outcome traits.

\section{Results}

\section{Timeline of UK-variant spreading}

The survey in sewage of the city of Nice showed that the UK-variant represented $2,6 \%, 8,3 \%$ and $79,1 \%$ of all identified SARS-CoV2 strains in December 2020, January 2021 and February 2021 respectively.

Consistent with these data, the random sequencing of positive PCR recorded from December 18th to 26th at Nice University hospital showed no identification of the UK-variant. This analysis was coordinated by the French National Center for Coronavirus (Institut des Agents Infectieux, CNR, HCL-Lyon) and was performed on $25 \%$ all the 73 positive patient's samples isolated during this time period.

Finally, a systematic screening PCR to identified latent variants was scheduled starting late January, on governmental recommendation. At this time, all positive PCR done at the Nice Hospital were screened. The rate of UK-variant during Aft1-F was $20 \%$ of all positive PCR, then $59 \%$ during Aft2-F.

Altogether, these data suggested that the UK-variant was absent in our area in December 2020, then increasingly spread in January 2021.

\section{Cohort profile of Covid-19 patients admitted in ED, overtime}

From December 1st to February 28th, 1247 patients were admitted for a suspected or diagnosed SARSCoV-2 infection (Elderly Care Facilities people not included), aged $66.1 \pm 17.9$ [17-102] years, $54.0 \%$ male gender. Six patients $(0.5 \%)$ died in the ED, while $n=731$ patients $(58.6 \%)$ were hospitalized in a medical ward and $n=109(8.7 \%)$ in ICU; the remaining, $n=401$ (32.1\%) were sent back home (ambulatory followup). Patients and clinical characteristics are reported (Table 1). 
Table 1

Patients and clinical characteristics of Covid-19 patients admitted in the emergency department from December 1 st to February 28 th

\begin{tabular}{|c|c|c|c|c|}
\hline & $\begin{array}{l}\text { December } \\
n=211\end{array}$ & $\begin{array}{l}\text { January } \\
n=457\end{array}$ & $\begin{array}{l}\text { February } \\
n=579\end{array}$ & $p$ \\
\hline Age years - mean (SD) & $68.0(17)$ & $67.3(17.7)$ & $64.4(18.3)^{\star \star * *}$ & 0.006 \\
\hline Patients over 65 years - n (\%) & $133(63)$ & $265(58)$ & $290(50) *, \star \star$ & 0.001 \\
\hline Male gender - n (\%) & $121(57)$ & $244(53)$ & $309(53)$ & ns \\
\hline Time 1st symptoms-ED - days (SD) & $6.4(4.3)$ & $7.1(4.9)$ & $7.5(4.4) *, \star \star$ & $<0.001$ \\
\hline \multicolumn{5}{|l|}{ Clinical signs } \\
\hline Respiratory rate/min. - mean (SD) & $22.0(7.1)$ & $23.8(9.3)$ & $24.4(8.4) *, \star \star$ & 0.001 \\
\hline Max Oxygen flow L/min - mean (SD) & $2.7(3.6)$ & $3.2(5.9)$ & $3.9(6.9)$ & $n s$ \\
\hline Max Temperature ${ }^{\circ} \mathrm{C}-$ mean (SD) & $37.3(0.9)$ & $37.3(0.9)$ & $37.4(1.2)$ & $n s$ \\
\hline Min SpO2 \% - mean (SD) & $91.9(6.1)$ & $91.9(7.6)$ & $91.8(7.2)$ & $n s$ \\
\hline NEWS-2 score - mean (SD) & $5.1(3.6)$ & $5.1(3.6)$ & $5.3(3.5)$ & $n s$ \\
\hline
\end{tabular}

Trends in patient's age (overall) and hospital location after ED (following the initial medical assessment) over these 3-month period are reported (Fig. 1).

Until February 22nd, we identified 29 hospitalized patients bearing the UK-variant. After pairing cases $(\mathrm{n}=$ 29) versus controls $(n=58)$ on age $(61.0 \pm 10.7$ vs $61.6 \pm 11.9$ years $)$ and gender $(51.7 \%$ male in each group), we compared time 1st symptoms-ED admission and the ED NEWS-2 score (cases vs controls) respectively: $7.3 \pm 3.5$ vs $6.8 \pm 3.9, p=0.424$ and $6.2 \pm 2.4$ vs $6.4 \pm 3.0, p=0.642$. Patients admitted in the ED required immediate ICU management in respectively $10.3 \%(3 / 29)$ and $12.0 \%(7 / 58), p=0.810$.

\section{Hospitalized patients in the Infectious Diseases and ICU departments : a three-periods assessment comparison}

The three a posteriori defined groups, i.e. Bef-F, Aft1-F and Aft2-F respectively, included 51,72 and 54 patients. Comparison of main data between groups are reported (Table 2). 
Table 2

Hospitalized patients in the Infectious Diseases and ICU departments : a three-periods assessment comparison. Bef-F: December 7th to 21st 2020, Aft1-F: January 24th to February 7th, Aft2-F: February 8th to $22 n d$

\begin{tabular}{|c|c|c|c|c|}
\hline Infectious Diseases patients & Bef-F $n=51$ & Aft1-F $n=72$ & Aft2-F n = 54 & $\mathbf{p}$ \\
\hline 1st symptoms - admission - days (SD) & $7.5(4.5)$ & $6.9(3.3)$ & $9.4(5.6) * \star$ & 0.01 \\
\hline Age - mean (SD) & $70.7(13.6)$ & $66.9(15.8)$ & $59.2(14.0) *, * \star$ & $<0.001$ \\
\hline Male - n (\%) & $33(65 \%)$ & $44(61 \%)$ & $31(57 \%)$ & 0.745 \\
\hline Diabetes - n (\%) & $17(33 \%)$ & $16(22 \%)$ & $12(22 \%)$ & 0.306 \\
\hline Hypertension - n (\%) & $20(39 \%)$ & $30(42 \%)$ & $15(28 \%)$ & 0.252 \\
\hline Obesity - n (\%) & $10(20 \%)$ & $18(25 \%)$ & $9(17 \%)$ & 0.503 \\
\hline Immunosuppression - n (\%) & $4(8 \%)$ & $5(7 \%)$ & $1(2 \%)$ & 0.341 \\
\hline No comorbidity - n (\%) & $8(16 \%)$ & $18(25 \%)$ & $23(42 \%) *, \star \star$ & 0.007 \\
\hline Charlson Index (CCl) - mean (SD) & $1.3(1.6)$ & $0.9(1.0)$ & $0.6(0.8) *, \star \star$ & 0.04 \\
\hline \multicolumn{5}{|l|}{ Clinical and radiological data } \\
\hline Respiratory Rate/min - mean (SD) & $25.0(6.1)$ & $26.9(5.8)$ & $24.9 \pm(5.9)$ & 0.051 \\
\hline Room air SpO2\% - mean (SD) & $90.5(7.2)$ & $91.2(5.6)$ & $92.1(4.6)$ & 0.335 \\
\hline CRP mg/L - mean (SD) & $94.3(85.9)$ & $108.8(77.7)$ & $94.4(68.2)$ & 0.316 \\
\hline CT-scan opacities ${ }^{\#}$ over $50 \%(n, \%)$ & $8(16 \%)$ & $10(14 \%)$ & $9(17 \%)$ & 0.951 \\
\hline ICU admission & $12(23 \%)$ & $19(26 \%)$ & $19(35 \%)$ & 0.374 \\
\hline ICU patients & Bef-F $n=13$ & Aft1-F $n=20$ & Aft2-F n = 22 & $\mathbf{p}$ \\
\hline Age (years) - mean (SD) & $68.4(10.8)$ & $64.5(10.7)$ & $60.6(12.5)$ & 0.154 \\
\hline Male - n (\%) & $9(69 \%)$ & $13(65 \%)$ & $16(72 \%)$ & 0.863 \\
\hline Mechanical ventilation - n (\%) & $7(54 \%)$ & $5(25 \%)$ & $10(45 \%)$ & 0.203 \\
\hline HFNC - n (\%) & $5(38 \%)$ & $14(70 \%)$ & $9(45 \%)$ & 0.140 \\
\hline SAPSII - mean (SD) & $40.6(18.1)$ & $35.5(15.3)$ & $40.0(19.9)$ & 0.566 \\
\hline Worst P/F - mean (SD) & $92(33)$ & $112(62)$ & $87(24)$ & 0.244 \\
\hline
\end{tabular}


At the time of analysis, some data were not analyzed because of missing points due to shortness of follow-up with patients still hospitalized (length of stay, mortality).

In patients under 65 years old hospitalized in ID department $(n=76)$, the rate of patients without any comorbidity rose from $21 \%$ (Bef-F) to $50 \%$ (Aft2-F), $p=0.029$.

\section{Discussion}

The occurrence of UK-variant at the end of 2020 is of major concern, because of the contagiousness issue $^{1,4}$ and therefore the risk of an increased number of patients requiring an ICU bed. Beyond the good organization of the flow of an increased number of patients, the question arises as to whether this lineage is also associated with a more severe clinical presentation, which could involve younger patients. Our results show that patients admitted in January and February 2021 in ED for a COVID-19 and therefore hospitalized for a COVID-19-related pneumonia are significantly younger with around $50 \%$ of them aged less than 65 years old. Our data strongly support the increased risk of SARS-CoV-2-related severe pneumonia in younger patients contemporaneously to the spread of the UK-variant in our aera.

The severity issue is less conclusive. The rate of ED patients admitted in ICU increases, but an increased clinical severity is not obvious, as neither the NEWS-2 score nor the main signs of clinical severity (oxygen requirement, respiratory rate) have changed over time. Even when UK-variant patients were paired on age and gender, no difference in term of early stage severity was evidenced. In the same way, patients hospitalized in the ID department didn't show a more severe inflammatory response or extended CT scan lung injuries at later times, i.e. after the spread of the UK-variant. Finally, for ICU patients, neither the severity score at admission (SAPSII) nor the depth of the respiratory distress seemed to increase by the variant. Altogether, there is no evidence to support a different and/or more severe clinical picture of the SARS-CoV-2-related pneumonia with the UK-variant.

The occurrence and severity of COVID-19 has been related to comorbidities like diabetes, hypertension or obesity ${ }^{6,7}$. Importantly, we provide herein data in favor of an increased ratio of healthy patients (i.e. without comorbidities) hospitalized for a SARS-CoV-2 related pneumonia associated to the spread of the UK-variant in the fifth-largest city of France. Combined to the patient's younger age, this brings several concerns. The most immediate is about the risk of having a pandemic evolving toward young and healthy populations, with even greater social consequences. Another consequence, supported by our data is the increase of patients with younger age/comorbidity free-related ICU requirement. So far, older patients with comorbidities have a greater risk of ICU admission ${ }^{8,9}$. The increase of younger people admitted in ED for a COVID-19, in our cohort, is associated with a trend of increased ICU ratio requirement afterward. The same trend was noticed in the ID department. As the severity is not obviously increased, a likely explanation is that physicians, after months of fighting against the pandemic with critical patients mainly over 65 years old, have been surprised to receive younger patients with severe pneumonia, leading possibly to a higher ICU demand. As the pandemic rises concerns about ICU being overwhelmed, such change in patient's profile brings new insights in the debate about ICU triage ${ }^{10,11}$. Furthermore, according 
to the country of interest, the vaccination strategy may have to be reconsidered in order to include a younger part of the population at risk of severe or critical COVID-19. Finally, the effect of the UK-variant on SARS-Cov-2-related mortality cannot be appropriately assessed here because, giving a mortality rate under $0.5 \%$, the required sample size needs to be thousands of patients ${ }^{2}$. The number of patients managed in our hospital and the duration of follow-up available do not allowed us to consider the final outcome for comparison between study periods.

Until today, reports of a relationship between SARS-CoV-2 genetic specificities among variants and clinical presentation are scarce. One variant with a deletion $(\Delta 382)$ in the open reading frame 8 have been associated to milder infections in Singapore ${ }^{12}$ and may have play a role in the very low case fatality rate in this country ${ }^{13}$. Spike mutation D614G that probably occurs in China before its diffusion in Europe is associated with a decreased age of COVID-19 patients possibly due to an increased viral load in younger patients ${ }^{14}$. The N501Y mutation is associated with adaptation to rodent, for instance mice ${ }^{15}$ and may increase SARS-CoV-2 Spike protein binding to ACE2 because of conformational changes, thus increasing its transmissibility ${ }^{1}$. The role of $\Delta 69 / \Delta 70$ on the spike protein is also potentially involved in the increased transmissibility ${ }^{16}$.

The spread of the UK-variant was found associated with a higher transmissibility as reported by others ${ }^{4}$. Since our data are mainly on hospitalized patients, the carriage of the UK-variant in a younger population bears further studies about the clinical fraction associated to the variant in the population ${ }^{17}$ outside the hospital. A puzzling question is why younger patients need now to be hospitalized, which was not the case before? The higher transmissibility of UK-variant is supposed to be similar among all age groups ${ }^{18}$. As of 26th of February, 60000 inhabitants of Alpes Maritimes had received 2 doses of vaccine. Increased herd immunity after vaccination of the old community and mortality during the two previous epidemic peaks in France that has affected the most fragile elderly may have independently contributed to the observation of a decreased number of patients over 75 year-old requiring hospitalization.

Our study has some limitations. First, data are retrospective. Then, data on the UK-variant spread results from analysis of sewage or selected hospital samples and not from a systematic survey. Data on hospitalized patients (variant versus "common" strain) are incomplete. Finally, patient's data were extracted from ED, ID and a medical ICU of the University hospital of Nice, but patients hospitalized in other hospitals from the ED were not included in the study, leading to incomplete analysis of involved patients. Our study do not allow to distinguish an effect of the UK-variant at the individual level, that is a higher probability to develop pneumonia after a contact with the virus due to the Spike protein RBD mutations, from a modification of virus diffusion in the South East of France population leading to a higher exposure of younger inhabitants which could be related to the virus itself or to a modification in non-pharmaceutical interventions adherence.

In summary, our data alerts on the consequences of the UK-variant spreading with younger and healthier patients requiring hospitalization for a SARS-CoV-2 pneumonia. Health system impact (with the risk of 
overwhelming ICU and medical units) as well as social consequences should be assessed in larger prospective studies.

\section{Method}

We designed an observational study to assess the clinical profile and outcome of patients infected with the SARS-CoV-2, before and during the spread of the UK-variant. Patients admitted in the Emergency department (ED), in the Infectious Diseases department (IDD) and the ICU at Nice University Hospital (Cote d'Azur University UCA), France, were part of this study. Data were extracted from the hospital electronic database $\left(n^{\circ} 410\right)$ of patient's record, registered on clinicaltrial.gov NCT04779021, also from the hospital Virology Lab and the Institut de Pharmacologie Moléculaire et Cellulaire, UMR7275 CNRS/UNS (for sewage samples analysis).

In the way to quickly characterize the clinical consequences of the spread of these new variants, we designed a three-step approach.

First step: Spread of the UK-variant in our area and among hospitalized patients. Data of the sequencing of SARS-CoV-2 identified from the sewage collector of the city of Nice (overall gathering) in December and January, and PCR screening from all positive samples analyzed at the hospital Virology Lab from newly admitted and diagnosed patients (starting late January 2021).

Second step: Concomitant epidemiology and clinical characteristics at hospital admission of COVID-19 overtime, by an evaluation over a three-month period, from December 1st 2020 to February 22nd 2021, of all patients admitted in the ED for COVID-19. Demographic data, clinical severity as recorded by the NEWS-2 scoring system and the follow-up (ward admission versus ambulatory follow-up). Patients coming from Elderly Care facilities clusters or transferred from another hospital with a SARS-CoV-2 positive PCR were not included in the study.

Third step: Clinical severity and follow-up of hospitalized patients for a SARS-CoV-2 pneumonia, in the IDD or ICU over three fortnights:

- before the spread of the UK-variant in our area,i.e. from December 7th to 21st 2020 (Bef-F),

- the two fortnights after starting a PCR screening from all positive samples analyzed at the hospital Virology Lab from newly admitted and diagnosed patients (late January 2021), i.e. from January 24th to February 7th and from February 8th to 22nd, 2021 respectively Aft1-F and Aft2-F)

Demographic data, clinical signs and severity were recorded by the NEWS-2 (ED), SAPS-2 and SOFA scores (ICU), CT-Scan findings and the follow-up were recorded and analyzed.

We assessed the UK-variant spread in sewage through the Nice wastewater plant, which treats the sewage for a population of 390,000 inhabitants. The daily incoming volume varied from 97,000 m3 (in December 15th, 2020) to 128,000 (January 21st, 2021), due to rain at the end of January. Sequencing 
followed the protocol of the Artic consortium (https://www.protocols.io/view/ncov-2019-sequencingprotocol-v3-locost-bh42j8ye), data being analyzed with their pipeline (https://artic.network/ncov2019/ncov2019-bioinformatics-sop.html). For each position associated with 20l/501Y.V1 lineage (https://cov-lineages.org/pangolin_tutorial.html) the fraction of 20l/501Y.V1 lineage reads relative to the total number of reads is assessed.

The virological assessment of positive PCR for SARS-CoV-2 variants at the Virology Lab was done as follow : SARS-CoV-2 positive samples were screened for the presence of the 20I/501Y.V1 lineage using either TaqPath COVID-19 RT-PCR (ThermoFisher, Illkirch-Graffenstaden, France) or ViroBOAR Spike 1.0 RT-PCR (Eurofins Biomnis, Lyon, France) kits, following manufacturer's instructions. Identification of the 20I/501Y.V1 variant was further verified by sequencing by sequencing a random set of samples in which the reliability of the RT-PCR screening tests was checked.. Briefly, Spike protein regions covering $\Delta 69 / 70$ and $\Delta 144$ deletions, as well as the N501Y substitution, were amplified through RT-PCR, and amplicons were sequenced by the Sanger method.

Patients who were hospitalized with an identification of the UK-variant at the Virology Lab (see upper) were paired (1:2) with patients hospitalized during the three first weeks of December (variant-free period), on age and gender. News-2 score, time 1st symptoms-ED admission and further hospital orientation (ICU versus medical ward) were the main evaluated criteria.

Data were expressed as mean \pm standard deviation SD. Comparisons are done using Chi-2, Fisher exacttest for categorical variables and Student-t test, Kruskall Wallis test and Man Whitney U-test for continuous variables comparisons, if required. All data were analyzed using SPSS software ${ }^{\circledR}$.

\section{Declarations}

\section{Data availability}

Data are available upon reasonable request

\section{Acknowledgments}

We thank Rainer Waldmann for fruitful discussions

Conflict of interest statement: none to declare

Contributions: JCou MC initiated the study. JCou JCon PB VG MC contributed to the study design. ED JL GR JD DC contributed to the implementation and supervision of the study. JCou JCon MC analysed the data and takes responsibility for the accuracy of the data analysis. JCou JCon PB VG MC drafted the manuscript. All authors read and approved the final manuscript.

\section{References}


1. Leung, K., Shum, M. H., Leung, G. M., Lam, T. T. \& Wu, J. T. Early transmissibility assessment of the N501Y mutant strains of SARS-CoV-2 in the United Kingdom, October to November 2020. Eurosurveillance 26, 2002106 (2021).

2. Challen, R. et al. Risk of mortality in patients infected with SARS-CoV-2 variant of concern 202012/1: matched cohort study. BMJ 372, n579 (2021).

3. Threat Assessment Brief: Rapid increase of a SARS-CoV-2 variant with multiple spike protein mutations observed in the United Kingdom. European Centre for Disease Prevention and Control https://www.ecdc.europa.eu/en/publications-data/threat-assessment-brief-rapid-increase-sars-cov-2variant-united-kingdom (2020).

4. Gaymard, A. et al. Early assessment of diffusion and possible expansion of SARS-CoV-2 Lineage 20I/501Y.V1 (B.1.1.7, variant of concern 202012/01) in France, January to March 2021. Euro Surveill. Bull. Eur. Sur Mal. Transm. Eur. Commun. Dis. Bull. 26, (2021).

5. COVID 19 point épidémiologique. https://www.santepubliquefrance.fr/dossiers/coronavirus-covid-19 (2021).

6. Zhou, F. et al. Clinical course and risk factors for mortality of adult inpatients with COVID-19 in Wuhan, China: a retrospective cohort study. Lancet Lond. Engl. 395, 1054-1062 (2020).

7. Yin, T., Li, Y., Ying, Y. \& Luo, Z. Prevalence of comorbidity in Chinese patients with COVID-19: systematic review and meta-analysis of risk factors. BMC Infect. Dis. 21, 200 (2021).

8. Tan, E., Song, J., Deane, A. M. \& Plummer, M. P. Global Impact of Coronavirus Disease 2019 Infection Requiring Admission to the ICU: A Systematic Review and Meta-analysis. Chest 159, 524-536 (2021).

9. Pijls, B. G. et al. Demographic risk factors for COVID-19 infection, severity, ICU admission and death: a meta-analysis of 59 studies. BMJ Open 11, e044640 (2021).

10. Wilkinson, D., Zohny, H., Kappes, A., Sinnott-Armstrong, W. \& Savulescu, J. Which factors should be included in triage? An online survey of the attitudes of the UK general public to pandemic triage dilemmas. BMJ Open 10, e045593 (2020).

11. Sprung, C. L. et al. Adult ICU Triage During the Coronavirus Disease 2019 Pandemic: Who Will Live and Who Will Die? Recommendations to Improve Survival. Crit. Care Med. 48, 1196-1202 (2020).

12. Young, B. E. et al. Effects of a major deletion in the SARS-CoV-2 genome on the severity of infection and the inflammatory response: an observational cohort study. Lancet Lond. Engl. 396, 603-611 (2020).

13. Kayano, T. \& Nishiura, H. A Comparison of Case Fatality Risk of COVID-19 between Singapore and Japan. J. Clin. Med. 9, (2020).

14. Volz, E. et al. Evaluating the Effects of SARS-CoV-2 Spike Mutation D614G on Transmissibility and Pathogenicity. Cell 184, 64-75.e11 (2021).

15. Gu, H. et al. Adaptation of SARS-CoV-2 in BALB/c mice for testing vaccine efficacy. Science 369 , 1603-1607 (2020). 
16. Neutralising antibodies in Spike mediated SARS-CoV-2 adaptation I medRxiv. https://www.medrxiv.org/content/10.1101/2020.12.05.20241927v3.full\#F6.

17. Davies, N. G. et al. Age-dependent effects in the transmission and control of COVID-19 epidemics. Nat. Med. 26, 1205-1211 (2020).

18. Investigation of novel SARS-CoV-2 variant: Variant of Concern 202012/01. GOV.UK https://www.gov.uk/government/publications/investigation-of-novel-sars-cov-2-variant-variant-ofconcern-20201201.

\section{Figures}

A

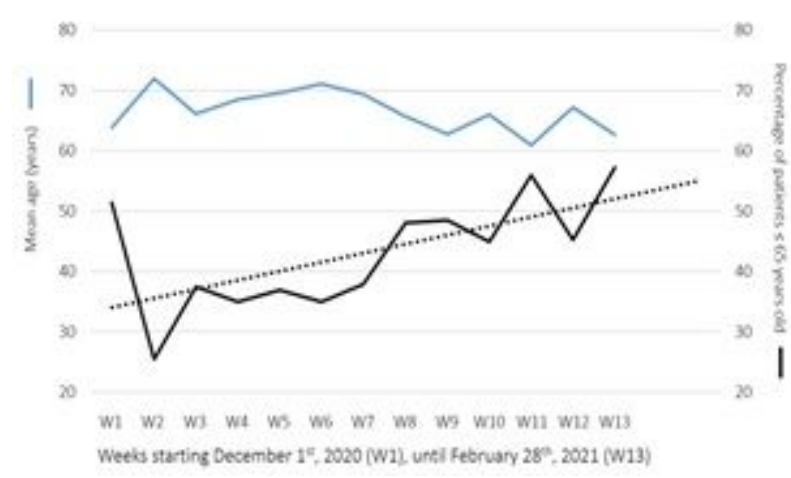

B

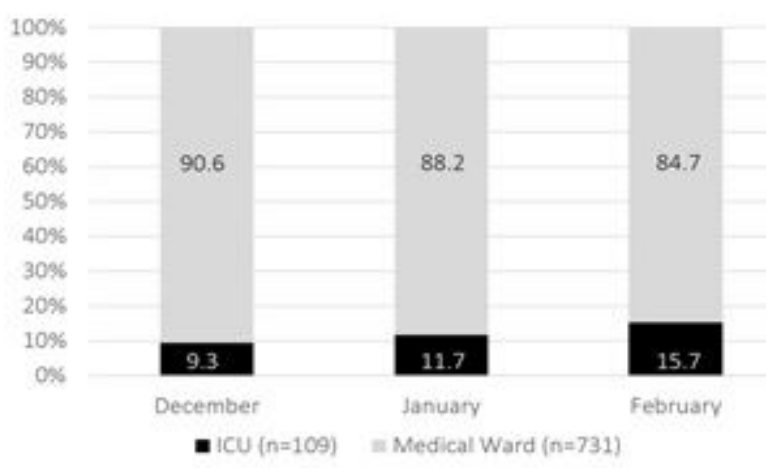

Figure 1

Age change and hospital admission after ED, over a 3 months-period. (A) mean age (blue line) and percentage of patients under 65 years old (black line, F-test 7.24, $p=0.021$ ) over weeks from December $1 \mathrm{st}, 2020, n=1247$. (B) ICU versus medical ward admissions (100\% normalized) for after ED hospitalization $(n=840)$ over the three months period, February versus December, $p=0.056$. 